\title{
Diagnóstico territorial de los recursos de apoyo social formal de atención a las mujeres desde los servicios sociales especializados
}

\author{
Maria Saray Florido Rubio \\ al016898@uji.es \\ M. ${ }^{a}$ Raquel Agost-Felip \\ ragost@uji.es
}

\section{Resumen}

La protección de la mujer en situación de vulnerabilidad incluye la existencia de recursos de apoyo social formal pertenecientes a la red de servicios sociales especializados. Si bien existen escasos estudios sobre la materia, desde el nuevo modelo de servicios sociales inclusivos que se está implementando, se considera necesario desarrollar el sistema basándose en evidencias empíricas (Uceda i Maza 2017). Así pues, el presente estudio tiene como objetivo realizar un diagnóstico territorial de los servicios sociales especializados de atención a la mujer en la provincia de Castellón, dada la escasez de estudios relacionados con esa materia. Para ello, se ha diseñado un estudio cualitativo, basado en la revisión de fuentes secundarias. Las fuentes consultadas han sido dos bases de datos (ARGOS y Mapificación de Centros Inclusivos). También se ha contado con información proporcionada desde la Conselleria de Igualdad y Políticas Inclusivas, sobre los centros acreditados existentes. Las variables estudiadas son: ámbito territorial (municipal y comarcal), tipo de centro, plazas existentes y modalidad de gestión.

Los resultados muestran los tipos de centros de servicios sociales especializados de atención a la mujer existentes: Infodona, centro de protección, centro 24 horas y vivienda tutelada. Se constata una distribución territorial de los recursos desigual, dado que se concentran en las zonas de mayor densidad urbana.

En conclusión, se observa la necesidad tanto de diseñar una distribución territorial más equitativa de recursos como de fortalecer la red de servicios sociales especializados de atención a la mujer y aumentar así los recursos de apoyo social formal hacia las mujeres en situación de mayor vulnerabilidad.

Palabras clave: apoyo social, mujer, servicios sociales especializados, servicios sociales inclusivos, territorio.

\section{Abstract}

The protection of women in vulnerable situations includes the existence of formal social support resources belonging to the specialized Social Services network. 
Although there are few studies on the subject, from the new model of inclusive Social Services that is being implemented, it is considered necessary to develop the system based on empirical evidence (Uceda i Maza 2017). Thus, this study aims to perform a territorial diagnosis of specialized social services for women in the province of Castellón, given that there are no studies related to this subject.

For this, a qualitative study has been designed, based on the review of secondary sources. The sources consulted were two databases (ARGOS and Mapping of Inclusive Centers). There has also been information provided by the Regional Ministry of Equality and Inclusive Policies, about the existing accredited centers. The variables studied are: territorial scope (municipal and regional), type of center, existing places and management modality.

The results show the types of Social Services centers specialized in women's care: Infodona, Protection Center, 24-hour Center, Sheltered Housing. There is an unequal territorial distribution of resources, given that they are concentrated in the areas with the highest urban density.

In conclusion, there is a need to design a more equitable territorial distribution of resources, as well as to strengthen the network of specialized Social Services for women, and thus increase the resources of formal social support for women in a situation of greater vulnerability.

Keywords: social support, women, specialized social services, inclusive social services, territory.

\section{Introducción}

Los servicios sociales, según el modelo de estructura de la comunidad Hombrados y Gómez de 1995 (Hombrados Mendieta 2013) (véase la ilustración 1), forman parte de la estructura de la comunidad, en concreto de la estructura formal, en la que se produce el apoyo social que contribuye a la cobertura de necesidades comunitarias, al igual que las prestaciones básicas y la provisión de recursos.

Ilustración 1. Estructura de la comunidad.

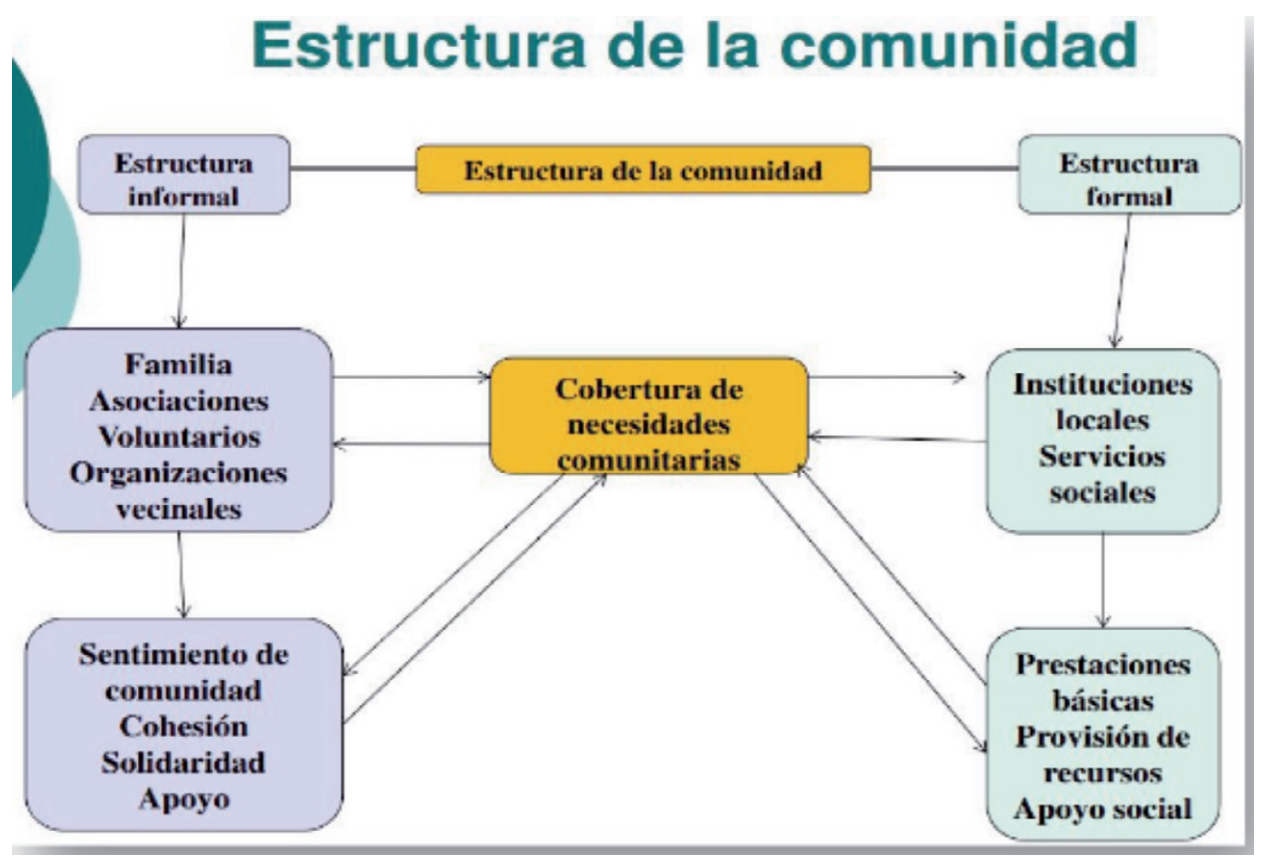


El actual sistema de servicios sociales se desarrolla a partir de la Constitución Española de 1978. A diferencia del sistema educativo o sanitario, en este caso no existe una ley de carácter estatal, sino que las comunidades autónomas han promulgado sus propias leyes. En el caso de la Comunidad Valenciana, se promulga inicialmente la Ley 5/1989, de 6 de julio, de Servicios Sociales de la Comunidad Valenciana, cuyo objetivo básico es la creación del sistema público de Servicios Sociales. Esta normativa se deroga con la entrada en vigor de la Ley 5/1997 de 25 de junio de la Generalitat Valenciana, por la cual se regula el sistema de servicios sociales en el ámbito de la Comunidad Valenciana, es en esta ley donde se regula el sistema de servicios sociales especializados de la mujer, sistema que se modifica en la Ley $3 / 2019$ de Servicios Sociales Inclusivos de la Comunidad Valenciana.

Los centros especializados son:

- Infodona (Orden 22/11/2004, Carta de Servicios de Infodona): es una red de centros de la Conselleria, cuyo objetivo es prestar servicios de atención e información personalizada a mujeres, grupos de mujeres, entidades y empresas de la Comunidad Valenciana a través de profesionales en cada uno de los diferentes centros. En estos centros se prestan diferentes servicios como son:

- Información sobre derechos, recursos, servicios públicos y normativa.

- Formación sobre creación de empresas, búsqueda de empleo y políticas de igualdad.

- Asesoramiento sobre planes de empresa, orientación profesional y formativa y orientación sociolaboral.

- Promoción de acciones de igualdad mediante la colaboración y orientación de proyectos y actividades relacionados con la igualdad de oportunidades.

- Centro Mujer 24 horas (artículo 59, Ley 7/2012, de 23 de noviembre, de la Generalitat, Integral contra la Violencia sobre la Mujer en el Ámbito de la Comunidad Valenciana): es un recurso público y gratuito. Su finalidad es procurar atención integral a las mujeres víctimas de malos tratos físicos o psíquicos, agresiones sexuales, abusos sexuales o acoso sexual.

- Centro de protección (artículo 59 de la Ley 7/2012, de 23 de noviembre, de la Generalitat, Integral contra la Violencia sobre la Mujer en el Ámbito de la Comunidad Valenciana): es un centro de recuperación integral, de carácter residencial, dirigido a mujeres víctimas de malos tratos, solas o acompañadas de sus hijas e hijos menores, con la finalidad de prestarles una ayuda psicológica y social, facilitándoles los medios básicos que les ayuden a superar la situación de violencia sufrida.

- Centro de acogida (Orden 17 de febrero de 2003): centro de alojamiento temporal y de atención integral a mujeres víctimas de malos tratos. La estancia oscila entre tres y seis meses, y es prorrogable.

- Vivienda tutelada (Orden 17 de febrero de 2003): es un piso en régimen de autogestión, destinado a la convivencia de mujeres que han sufrido malos tratos, acompañadas de menores a su cargo, y que necesitan acogimiento de carácter temporal para poder integrarse con autonomía en su entorno. La estancia es de aproximadamente de un año.

Tras la crisis económica, que se inició a finales del $2006,{ }^{1}$ se produjo un cambio en la economía familiar y en la situación laboral de España. En el 2008, la cuarta parte de los

\footnotetext{
${ }^{1}$ Datos del Barómetro social.
} 
hogares había experimentado un descenso notable de sus ingresos y casi uno de cada tres llegaba con dificultades a fin de mes. Entre 2008-2009 se produce una brusca pérdida de 1,4 millones de puestos de trabajo (Colectivo IOE 2010; 2011).

Este y otros motivos son los que hacen necesaria la promulgación de una nueva ley de Servicios Sociales; además, la ley de 1997 es todavía una ley de primera generación, cuando en otras autonomías están en vigor leyes de tercera generación. ${ }^{2}$ Esta situación se refleja en el índice DEC ${ }^{3}$ del año 2017 y nos indica que la Comunidad Valenciana, junto con Canarias, son las que peor puntuación han obtenido en el desarrollo de los servicios sociales, si bien se ha mejorado frente a evaluaciones anteriores (Asociación de Directoras y Gerentes en Servicios Sociales 2017).

En la actualidad, se ha promulgado una nueva Ley 3/2019 de Servicios Sociales Inclusivos de la Comunidad Valenciana. Los motivos que fundamentan este texto recogen la necesidad de introducir el derecho subjetivo, donde se reconozca y garantice la prestación a todas las personas que acrediten necesidad, y no condicionar la atención a la disponibilidad presupuestaria. También se recoge regular el sistema para adaptarlo a las nuevas realidades sociales y mejorar su ordenamiento orientado a una mejor estructura funcional y territorial, que pueda garantizar tanto la igualdad de oportunidades como la equidad territorial.

Los servicios sociales especializados en la mujer, en su mayoría, están desbordados por las demandas de mujeres víctimas de violencia de género. En muchos de estos casos, son mujeres en situación de exclusión social, que requieren de gran apoyo no solo institucional, sino familiar y social. Se entiende como factores de exclusión social, entre otros, el nivel socioeconómico, la situación laboral, si poseen alguna discapacidad funcional, etc. ${ }^{4}$ La violencia contra las mujeres es uno de los problemas que afectan a la sociedad actual. Durante el año 2017 se registraron un total de ocho muertes en la Comunidad Valenciana (Consejo General del Poder Judicial 2017) y, según el informe de la Red de Centros de Mujer (2017), se atendió en materia de violencia de género a un total de 740 mujeres solo en la provincia de Castellón, número más que significativo de mujeres en situación de dependencia emocional, social y económica, que se atienden desde el sistema de servicios sociales.

Cuando se habla del sistema de servicios sociales, no solo nos referimos a la atención del personal técnico, sino que dicha atención se realice en centros próximos o bien que no requiera de un desplazamiento demasiado largo. Por ello, se plantea la necesidad de realizar un diagnóstico territorial de los recursos y centros existentes, para conocer cuál es su distribución. No se han encontrado estudios que aborden la perspectiva territorial de la atención de los Servicios Sociales especializados, a excepción de un estudio reciente coordinado por Martínez Martínez y Uceda i Maza (2017) referido al análisis territorial de los servicios sociales en la provincia de Valencia, donde se incluye un análisis de los servicios sociales para la mujer (Canet Benavent y Garrigós i Santos 2017).

Así pues, el objetivo de este trabajo es conocer cuál es la distribución territorial de los servicios sociales especializados dirigidos a la mujer en la provincia de Castellón. Para ello se plantean los siguientes objetivos específicos: conocer el tipo de centros de servicios sociales especializados en la mujer que existen en la provincia de Castellón, conocer la modalidad de gestión de estos centros (pública, privada) y conocer el número de las plazas existentes en cada uno de ellos. Este análisis, a su vez, permitirá localizar

\footnotetext{
2 Estas leyes son las que incorporan el derecho subjetivo y una cartera de servicios.

3 Índice de Desarrollo de los Servicios Sociales (índice DEC).

4 Datos del Centro Mujer 24 horas de la Comunidad Valenciana, del año 2017.
} 
los recursos en el mapa de la provincia de Castellón para conocer su distribución territorial.

\section{Método}

Este estudio tiene como objeto el conocer el estado actual de los servicios sociales especializados de atención a la mujer en la provincia de Castellón. Para ello, se ha diseñado un estudio cualitativo donde se han utilizado fuentes secundarias de información y se ha adaptado la metodología utilizada por Canet Benavent y Garrigós i Santos (2017). Se han consultado tres bases de datos generalistas públicas, dos de ellas de acceso abierto a través de la web, donde se recogen los recursos objeto de estudio.

Para este diagnóstico territorial, se han analizado los recursos especializados de atención a la mujer, sea cual sea la condición de exclusión social en la que se encuentre.

Las bases de datos utilizadas han sido: el portal de información ARGOS, ${ }^{5}$ la Mapificación de Centros Inclusivos de la Vicepresidencia y Conselleria de Igualdad y Políticas Inclusivas ${ }^{6}$ y el Registro y Autorización de Funcionamiento de los Servicios y Centros de Acción Social, en la Comunidad Valenciana (Decreto 91/2002). Esta información se ha completado con las páginas web de los municipios de la provincia de Castellón y con la base de datos de la Fundación Isonomía (2018).

Las variables de estudio son: el tipo de centro, la modalidad de gestión, el número de plazas ofertadas, y el municipio donde se encuentra el centro:

1. Tipo de centro: según la Orden de 17 febrero de 2003, de Conselleria de Bienestar Social, sobre condiciones y requisitos para la autorización de los centros especializados para mujeres en situación de riesgo social, los centros son: Infodona, centro mujer 24 horas, centro de protección, centro de acogida y vivienda tutelada.

2. Gestión: hace referencia a la entidad que lleva el mantenimiento del centro. Este puede estar gestionado por la Generalitat Valenciana, por el municipio (carácter local) o por una entidad privada (tercer sector o empresa), según lo referido en la Ley $7 / 1985$, de 2 de abril, Reguladora de Bases de Régimen Local. ${ }^{7}$

3. Número de plazas: hace referencia a la cantidad de plazas existentes en los centros de carácter residencias dirigidos a la mujer.

4. Localización: hace referencia al municipio y comarca donde se encuentra el centro (Alto Mijares, Alto Palancia, Baix Maestrat, Els Ports, Plana Alta, Plana Baixa, l'Alcalatén o Alt Maestrat).

\footnotetext{
${ }^{5}$ ARGOS: portal de información de la Dirección de Análisis y Políticas Públicas de la Presidencia de la Generalitat, por medio del cual se pone al alcance de los ciudadanos y de todo aquel que busque información especializada diferentes recursos informáticos y documentales de que dispone la Dirección General, como son las bases de datos electorales y municipales, dosieres temáticos, repertorios bibliográficos, etc. elaborados y gestionados por este departamento

6 Datos obrantes en el Registro General de los titulares de actividades, y de los servicios y centros de acción social, creado en virtud del Decreto 91/2002 de 30 de mayo, del Gobierno Valenciano.

${ }^{7}$ Es el Estado el que podrá delegar la gestión de los servicios sociales en sus comunidades y también en los municipios. En primera instancia es la comunidad la que tiene la potestad y son los municipios de más de 20000 habitantes los que podrán ejercer dichas competencias, también delegando en otros municipios si el Estado lo estima oportuno.
} 


\section{Resultados}

De acuerdo con las variables seleccionadas, los resultados se presentan en el cuadro 1 y la representación territorial está recogida en el mapa 1.

Los resultados obtenidos son los siguientes:

1. Tipo de centro. Se registran cuatro tipos: Infodona, centro mujer 24 horas, centro de acogida y vivienda tutelada. De estos dos últimos, por motivos de protección a la víctima, no se ha facilitado la localización, por lo que no aparecen en el mapa de resultados (mapa 1 ).

2. Gestión. Todos los recursos son gestionados por la Generalitat Valenciana.

3. Número de plazas. El centro de acogida cuenta con 35 plazas y las dos viviendas tuteladas cuentan con 14 plazas en total.

4. Localización. Existen 9 municipios con recursos especializados en la provincia de los 135 que existen. Según el análisis comarcal, los resultados encontrados son:

- Alto Palancia: solo la localidad de Segorbe cuenta con un centro especializado para la mujer, Infodona.

- Alto Mijares: no hay ninguna población con servicios sociales especializados para la mujer.

- Plana Baixa: las localidades de La Vall D'Uixó y de Vila-real cuentan con un centro especializado para la mujer, Infodona.

- L'Alcalaten: en esta comarca no existe ningún municipio donde existan servicios sociales especializados para la mujer.

- Plana Alta: dos municipios albergan centros de servicios sociales especializados para la mujer, Almazora y Castellón de la Plana.

- Alt Maestrat: se localiza allí un centro Infodona, ubicado en el municipio de Villafranca.

- Els Ports: la localidad de Morella cuenta con un centro Infodona.

- El Baix Maestrat: dos municipios cuentan con centros Infodona, Benicarló y Vinaroz.

La población de la provincia según el INE (2016) es de 579245 habitantes, de los que 290250 son mujeres y 286580 son hombres.

Tabla 1

Resultados de la localización de los recursos en la provincia de Castellón 2017.

\begin{tabular}{|l|l|l|l|c|c|}
\hline \multicolumn{1}{|c|}{$\begin{array}{c}\text { Comarca } \\
\text { Alto Palancia }\end{array}$} & $\begin{array}{l}\text { Población } \\
\text { Segorbe }\end{array}$ & $\begin{array}{l}\text { Habitantes } \\
9005\end{array}$ & $\begin{array}{c}\text { Centro } \\
\text { Infodona }\end{array}$ & $\begin{array}{r}\text { Gestión } \\
\text { Generalitat } \\
\text { Valenciana (GV) }\end{array}$ & Plazas \\
\hline Plana Baixa & Vall D'Uixó & 31819 & $\begin{array}{l}\text { Infodona } \\
\text { Vila-real }\end{array}$ & 50252 & Infodona \\
Alt Maestrat & Villafranca & 2289 & Infodona & GV \\
\hline Plana Alta & Almazora & 25635 & Infodona & GV \\
& Castellón & 170990 & Infodona & GV \\
& de la Plana & & Centro M. & GV & \\
& & & 24horas & \\
\hline
\end{tabular}




\begin{tabular}{|c|c|c|c|c|c|}
\hline Els Ports & Morella & 2504 & Infodona & GV & \\
\hline \multirow{2}{*}{$\begin{array}{l}\text { Baix } \\
\text { Maestrat }\end{array}$} & Benicarló & 26486 & Infodona & GV & \\
\hline & Vinaroz & 28290 & Infodona & GV & \\
\hline Se desconoce & No datos & No datos & $\begin{array}{l}\text { Centro de } \\
\text { acogida }\end{array}$ & - & 34 \\
\hline Se desconoce & No datos & No datos & $\begin{array}{l}\text { Vivienda } \\
\text { tutelada }\end{array}$ & - & 14 \\
\hline
\end{tabular}

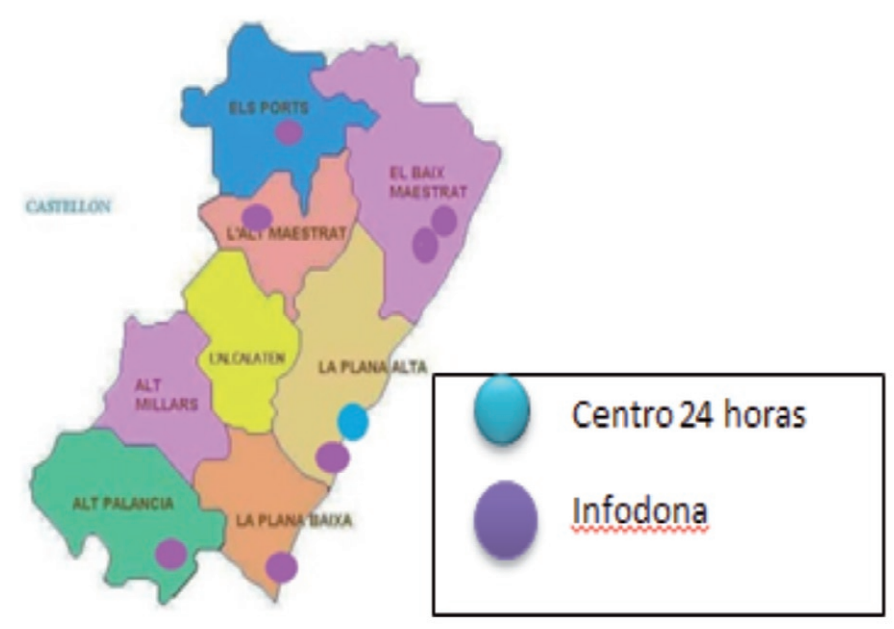

Figura 1. Localización según tipo de centro.

\section{Discusión y conclusiones}

La distribución de los servicios sociales está relacionada con la población: a mayor densidad demográfica, mayor variedad de recursos. Este análisis constata la inequidad en el acceso y en la distribución territorial de los servicios sociales especializados dirigidos a la población más vulnerable. Al igual que otros estudios realizados en la Comunidad Valenciana (Escribano Pizarro, Herráiz Lizan y Serrano Lara 2017), es en las zonas urbanas donde se atiende a todos los sectores de la población y donde se encuentra la mayoría de equipamientos; en las zonas rurales no se ofrece a la población todos los recursos especializados.

La gestión de los centros es pública y corresponde a la Generalitat Valenciana. Cada vez existen más municipios que cuentan en sus plantillas con la figura profesional del agente de igualdad, como demuestra la lista extraída por la red de agentes de igualdad, según el Institut de la Dona. ${ }^{8}$ Este es un perfil profesional que promueve la gestión eficaz e igualitaria de los derechos, recursos y deberes de toda la ciudadanía.

\footnotetext{
${ }^{8}$ Véanse los datos de Vicepresidencia y Conselleria de Igualdad y Políticas Inclusivas.
} 
Así pues, los resultados muestran la inequidad territorial de los servicios sociales especializados de atención a la mujer, así como la falta de más tipos de centros que puedan atender a las mujeres. Según los objetivos del estudio, se constata la falta de centros que puedan atender de manera integral a la mujer. Existen nueve centros Infodona que son de carácter informacional, el centro mujer 24 horas se encuentra en la capital de provincia y solo existen tres centros de carácter residencial (un centro de protección y dos viviendas tuteladas).

El número de plazas ofertadas en el centro de acogida y las viviendas tuteladas es escaso. Según los datos obtenidos, en la provincia de Castellón existen 48 plazas para recursos especializados de atención residencial y, según datos del Consejo General de Poder Judicial, solo en la provincia de Castellón en el año 2017 se tramitaron $426^{9}$ órdenes de protección, con sus medidas correspondientes, siendo una de ellas el acceso a una vivienda digna. Otro dato que corrobora la falta de recursos es el que refleja el índice DEC (2017): la Comunidad Valenciana en número de plazas ha obtenido la nota más baja (un 0), si bien se están haciendo esfuerzos para mejorar el índice DEC.

La localización territorial permite visibilizar la desigual distribución de los recursos entre las zonas de interior (menos pobladas) y las zonas costeras (más pobladas). Este hecho también agrava la situación de las mujeres del interior que deseen acceder a ciertos recursos y se generan problemas de movilidad. En las comarcas del Alto Mijares y de l'Alcalatén no se encuentra ningún centro de atención especializada. Una de las limitaciones del estudio está relacionada con dificultades existentes en encontrar la información en una base de datos actualizada, puesto que se ha tenido que recurrir a diferentes fuentes que recogen los datos de Conselleria, dado que la información no está centralizada. Para futuros trabajos se propone completar esta información con el resto de recursos de atención a la mujer y promoción de la igualdad, bien vinculados a servicios sociales generales o a áreas municipales específicas.

Para finalizar, se considera necesario implementar una agenda que permita diseñar una red equitativa de centros de servicios sociales especializados dirigidos a la atención de la mujer, así como incrementar los programas y servicios que trabajen para prevenir y erradicar la violencia de género, puesto que, a día de hoy, las mujeres siguen muriendo a manos de sus parejas (véase la gráfica 1). Para ello, también se propone mejorar la coordinación existente entre los diferentes sistemas de protección y de prevención, como son las fuerzas y cuerpos de seguridad del Estado, sanidad y servicios sociales, y evitar que la víctima acarree con una doble victimización, como es el caso de las mujeres maltratadas. En definitiva, continuar trabajando para conseguir comunidades más inclusivas y competentes y trabajar por la igualdad de género tal y como se promueve en los Objetivos de Desarrollo Sostenible (ONU 2015).

\footnotetext{
${ }^{9}$ Consultar web del Poder Judicial.
} 


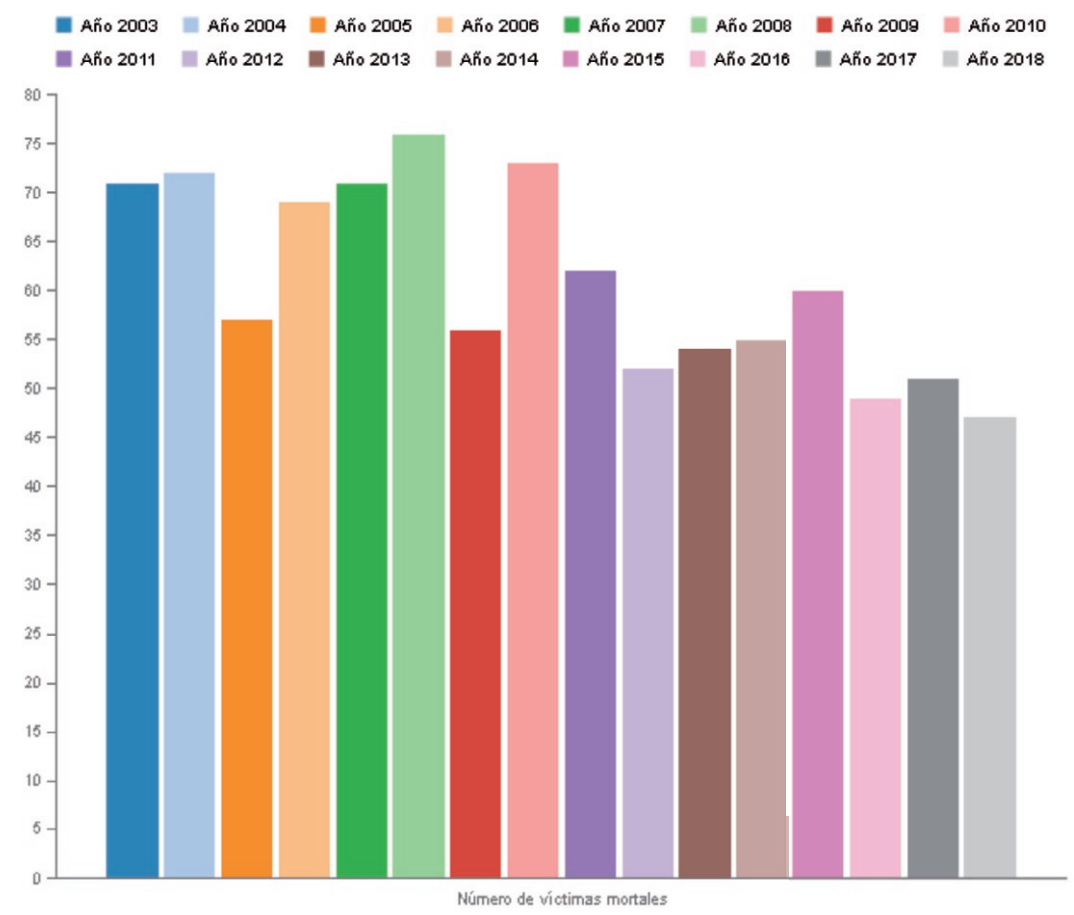

Gráfico 1. Datos extraídos del portal estadístico de la Delegación del Gobierno para la Violencia de Género. Del año 2003 al 2018.

\section{Referencias bibliográficas}

Asociación de Directoras y Gerentes en Servicios Sociales. 2017. Índice DEC 2017. Recuperado de: https://www.consaludmental.org/publicaciones/Indice-DEC2017.pdf [Consultado el 2 de agosto de 2019].

Canet Benavent, Encarna y Pere Joan Garrigós i Santos. 2017. "Los Servicios Sociales para la mujer». En Los Servicios Sociales en la provincia de Valencia. Análisis territorial y estado de la cuestión, coord. Lucía Martínez Martínez y Francesc Xavier Uceda i Maza (191-2012). Valencia: Publicacions de la Universitat de València.

Colectivo IOE. 2010. Efectos sociales de la crisis. Una valoración a partir del Barómetro social de España. Recuperado de:

http://www.barometrosocial.es/archivos/BSE_2008.pdf?9f0716 [Consultado el 2 de agosto de 2019].

-. 2011. Una evaluación del bienestar social. Recuperado de: www.barometrosocial.es/archivos/BSE_2009.pdf?9f0716 [Consultado el 2 de agosto de 2019].

Consejo General del Poder Judicial. 2017. La violencia sobre la mujer en la estadística judicial - Anual 2017. Recuperado de:

http://www.poderjudicial.es/cgpj/es/Temas/Violencia-domestica-y-degenero/Actividad-del-Observatorio/Datos-estadisticos/La-violencia-sobre-la-mujeren-la-estadistica-judicial---Anual-2017

Constitución Española. Boletín Oficial del Estado, 29 de diciembre de 1978, número 311.

Decreto 91/2002, de 30 de mayo, del Gobierno Valenciano, sobre Registro de los Titulares de Actividades de Acción Social, y de Registro y Autorización de Funcionamiento de los Servicios y Centros de Acción Social, en la Comunidad Valenciana. 
Escribano Pizarro, Jaime, Cristina Herráiz Lizan y Jose Javier Serrano Lara. 2017. «La organización territorial de los Servicios Sociales especializados en la Provincia de Valencia. Un (des)ajuste entre la oferta y la demanda». En Los Servicios Sociales en la provincia de Valencia, coord. Lucía Martínez Martínez y Francesc Xavier Uceda i Maza (287-334). Valencia: Publicacions de la Universitat de València.

Instituto Nacional de Estadística. 2016. Cifras oficiales de población.

Fundación Isonomía. 2018. Red Isonomía. Recuperado de: http://isonomia.uji.es/redisonomia/.

Hombrados Mendieta, María Isabel. 2013. Manual de Psicología Comunitaria. Madrid: Síntesis.

Ley $7 / 1985$, de 2 de abril, Reguladora de Bases de Régimen Local. Boletín Oficial del Estado número 80, Madrid, 3 de abril de 1985.

Ley 5/1989, de 6 de julio, de Servicios Sociales de la Comunidad Valenciana. Diari Oficial de la Generalitat Valenciana, número 1105, Valencia, 12 de julio de 1989.

Ley $5 / 1997$, de 25 de junio, de la Generalitat Valenciana, por la que se regula el Sistema de Servicios Sociales en el Ámbito de la Comunidad Valenciana. Diari Oficial de la Generalitat Valenciana, número 3028, Valencia, 4 de julio de 1997.

Ley $7 / 2012$, de 23 de noviembre, de la Generalitat, Integral contra la Violencia sobre la Mujer en el Ámbito de la Comunidad Valenciana. Diari Oficial de la Generalitat Valenciana, núm. 6912, Valencia, 28 de noviembre de 2012.

Ley $3 / 2019$, de 18 de febrero, de la Generalitat, de Servicios Sociales Inclusivos de la Comunidad Valenciana [2019/1653].

Martínez Martínez, Lucía y Francesc Xavier Uceda i Maza. 2017. «Los Servicios Sociales en la Comunidad Valenciana». En Los Servicios Sociales en la provincia de Valencia. Análisis territorial y estado de la cuestión, coord. Lucía Martínez Martínez y Francesc Xavier Uceda i Maza (31-65). Valencia: Publicacions de la Universitat de València.

Ministerio de Sanidad, S. S. 2017. Violencia de género. Recuperado de: http://www.violenciagenero.igualdad.mpr.gob.es/violenciaEnCifras/victimasMortale s/fichaMujeres/pdf/VMortales_31_12_2017

ONU. 2015. Agenda 2030. Objetivos del Desarrollo Sostenible (ODS) Recuperado de: https://www.un.org/sustainabledevelopment/es/2015/09/la-asamblea-generaladopta-la-agenda-2030-para-el-desarrollo-sostenible/ [Consultado el 2 de agosto de 2019].

Orden de 17 de febrero de 2003, de la Conselleria de Bienestar Social, sobre condiciones y requisitos para la autorización de los Centros Especializados para mujeres en situación de riesgo social, por la que se desarrolla el Decreto 91/2002, de 30 de mayo, del Gobierno Valenciano, sobre Registro de los Titulares de Actividades de Acción Social, y de Registro y Autorización de Funcionamiento de los Servicios y Centros de Acción Social, en la Comunidad Valenciana

Orden de 22 de noviembre de 2004, de la Conselleria de Bienestar Social, por la que se aprueba la Carta de Servicios de Infodona. Diari Oficial dela Generalitat Valenciana, número 4910, Valencia 24 de diciembre de 2004

Red de Centros de Mujer. 2017. Informe anual 2017. Recuperado de: http://www.sinmaltrato.gva.es/documents/454751/456556/CENTROS+MUJER+24+ HORAS+A\%C3\%910+2017/2741f81a-fe54-4186-b54e-73a7497e76c4 [Consultado el 2 de agosto de 2019].

Uceda i Maza, Francesc Xavier. 2017. «Una agenda Valenciana de transfomació social: un nou model valencià». Revista Valenciana d'Estudis Autonòmics, 62: 89-122. 